\title{
Prevalência e fatores associados à presença de medicamentos vencidos em estoques caseiros
}

\section{Prevalence and factors associated to the presence of expired medications in households}

Máyra Rodrigues Fernandes' (D), Rafaela Silva Rocha' (D), Isabella Ribeiro Silva' (D), Roberta Carvalho Figueiredo' (D) , André Oliveira Baldoni ${ }^{1}$ (D)

'Universidade Federal de São João del-Rei (UFSJ), Campus Centro-Oeste Dona Lindu - Divinópolis (MG), Brasil.

Como citar: Fernandes MR, Rocha RS, Silva IR, Figueiredo RC, Baldoni AO. Prevalência e fatores associados à presença de medicamentos vencidos em estoques caseiros. Cad Saúde Colet, 2020;28(3):390-399. https://doi.org/10.1590/1414$462 \times 202028030535$

\section{Resumo}

Introdução: Medicamentos vencidos em farmácias caseiras são potenciais agentes causadores de danos à saúde humana e ao meio ambiente. Neste sentido, são essenciais estudos que subsidiem políticas de intervenção para minimização dos riscos. Objetivo: Estimar a prevalência e os fatores associados aos medicamentos vencidos em estoques caseiros. Método: $O$ estudo transversal foi realizado por meio de entrevistas nas residências dos usuários da atenção primária à saúde (APS) de uma cidade do centro-oeste mineiro, Brasil. As estimativas de prevalência e suas associações foram realizadas por meio de modelos lineares generalizados (MLGs). Resultados: Nos 423 estoques caseiros, foram encontrados 4.203 medicamentos, com uma média de 10 medicamentos/residência ( $D P=5,87$ ). A prevalência de residências com medicamentos vencidos foi de $45,4 \%$, sendo os antimicrobianos e analgésicos os mais prevalentes. A presença de medicamentos vencidos está associada à presença de medicamentos isentos de prescrição (MIPs) e antimicrobianos nas residências e ao comportamento de guardar o medicamento em desuso em seu estoque caseiro. Conclusão: Aproximadamente metade das residências possui medicamentos vencidos, e a presença deles está associada à classe terapêutica e ao comportamento do usuário.

Palavras-chave: armazenamento de medicamentos; farmacoepidemiologia; prazo de validade de medicamentos; sistema único de saúde.

\begin{abstract}
Background: The storage of expired medications at home can cause potential damage to human health and the environment. Thus, studies that support intervention policies to minimize risks are essential. Objective: To estimate the prevalence and factors associated with storage of expired medications at home. Method: The cross-sectional study was conducted through interviews in the homes of Primary Health Care (PHC) users of a city in the center-west of Minas Gerais, Brazil. Prevalence estimates and their associations were performed using generalized linear models (MLGs). Results: We found 4,203 medications stored in 423 homes, with an average of 10 medication/residence ( $S D=5.87)$. The prevalence of homes with expired medications was $45.4 \%$, with antimicrobials and analgesics being the most prevalent. The presence of expired medications is associated with the presence of Over the Counter drugs (OTC) and antimicrobials at homes, and the behavior of keeping the unused drug in their home pharmacy. Conclusion: Almost half of the homes have expired medications. And the presence of these drugs is associated with the therapeutic class and the behavior of the PHC user.
\end{abstract}

Keywords: drug storage; pharmacoepidemiology; medication expiration; unified health system.

Trabalho realizado na Universidade Federal de São João Del-Rei (UFSJ), no Campus Centro-Oeste Dona Lindu (CCO) Divinópolis (MG), Brasil.

Correspondência: André Oliveira Baldoni. E-mail: andrebaldoni@ufsj.edu.br

Fonte de financiamento: Coordenação de Aperfeiçoamento de Pessoal de Nível Superior - Brasil (CAPES) - Código de Financiamento 001.

Conflito de interesses: nada a declarar.

Recebido em: Nov 08, 2018. Aprovado em: Set. 12, 2019

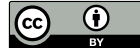

Este é um artigo publicado em acesso aberto (Open Access) sob a licença Creative Commons Attribution, que permite uso, distribuição e reprodução em qualquer meio, sem restrições desde que o trabalho original seja corretamente citado. 


\section{INTRODUÇÃO}

Os medicamentos representam um dos maiores e mais crescentes custos para os sistemas de saúde em todo o mundo ${ }^{1}$. O consumo de medicamentos no Brasil segue a tendência mundial, deixando o país entre os 10 maiores mercados farmacêuticos do mundo ${ }^{2}$. $\mathrm{O}$ acesso gratuito a medicamentos é um dever do Estado brasileiro, que incorporou, entre seus princípios e diretrizes constitucionais, a garantia da assistência terapêutica integral, incluindo assistência farmacêutica no âmbito do Sistema Único de Saúde (SUS) ${ }^{3}$. Assim, os gastos públicos com medicamentos são crescentes e constituem o segundo maior item de despesa dos sistemas de atenção à saúde. No período entre 2013 e 2014, o orçamento do Ministério da Saúde destinado à assistência farmacêutica passou de $R \$ 1,9$ bilhão para $R \$ 12,4$ bilhões, com investimento superior a $\mathrm{R} \$ 80$ bilhões na área no período ${ }^{4}$.

Mesmo com o elevado investimento para aumentar o acesso aos medicamentos, dados da Organização Mundial da Saúde ${ }^{5}$ indicam que mais da metade de todos os medicamentos utilizados mundialmente é prescrita, dispensada ou utilizada de forma incorreta. Assim sendo, a ampliação do acesso não garante o uso correto e racional do medicamento. Neste sentido, é importante destacar que a não adesão ao tratamento farmacológico é um dos principais problemas farmacoterapêuticos existentes no Brasil e em outros paíse ${ }^{6-8}$, gerando consequências clínicas, humanísticas e econômicas que oneram o sistema de saúde de forma desnecessária, exemplificado pelo acúmulo e pelo vencimento de medicamentos nos domicílios $s^{6,9}$.

Os medicamentos vencidos constituem um risco considerável de uso irracional, podendo resultar em intoxicações, efeitos indesejáveis e falta de efetividade na terapia medicamentosa ${ }^{10}$. Associado a isso, há o descarte incorreto desses medicamentos no meio ambiente, que, mesmo quando em baixos níveis de concentração, são suficientes para causar impactos ambientais. Alguns dados de alteração de ecossistemas por produtos farmacêuticos são relatados na literatura, como o aumento da resistência bacteriana e possíveis modificações genéticas em seres humanos e em animais marinhos ${ }^{11}$. Desta forma, a presença de medicamentos não utilizados e vencidos tem sido um problema de saúde pública no Brasil e no mundo ${ }^{10-12}$.

Diante disso e da escassez de dados sobre o desperdício de medicamentos contidos nas residências de usuários da atenção primária à saúde $(\mathrm{APS})^{13}$, o presente estudo teve como objetivo estimar a prevalência e os fatores associados aos medicamentos vencidos nos estoques caseiros de usuários da APS do SUS de uma cidade do centro-oeste mineiro, Brasil.

\section{MÉTODO}

\section{Local e população do estudo}

Estudo transversal realizado com indivíduos atendidos em unidades de atenção primária do município de Divinópolis, em Minas Gerais, Brasil, cuja população consiste em 217.404 mil habitantes ${ }^{14}$. O município possui 34 centros de APS e cinco farmácias públicas.

Foram incluídos indivíduos com 18 anos ou mais, atendidos em unidades da APS e usuários de farmácia pública do município. As unidades de saúde da atenção primária localizadas em zona rural e com alta taxa de violência urbana foram excluídas do sorteio por limitar a viabilização do estudo. Desta maneira, das 34 unidades de saúde, 15 foram sorteadas aleatoriamente para garantir diversidade na amostra e, por fim, alcançar o espaço amostral necessário. A estratégia amostral considerou o tamanho da população atendida pelas unidades de saúde para que todas tivessem a mesma probabilidade de serem sorteadas. Para efeito de cálculo amostral, foram considerados os seguintes parâmetros: a) prevalência de $50 \%$, dada a escassez de evidências científicas sobre a prevalência de medicamentos vencidos em domicílios; b) precisão de 5\%; c) nível confiança de 95\%; e d) 10\% de perdas. Sendo assim, a amostra calculada foi de 423 usuários da APS.

\section{Seleção dos participantes e coleta de dados}

Os indivíduos foram convidados a participar do estudo quando estavam nos centros de saúde sorteados, antes ou depois do atendimento. Nesse momento, o entrevistador explicava o objetivo, a análise do perfil de uso e o armazenamento de medicamentos no domicílio. 
Caso o usuário da atenção primária do SUS aceitasse, o entrevistador registrava o endereço e o telefone para posterior visita domiciliar. Foi realizada apenas uma entrevista por domicílio.

As entrevistas foram realizadas no período da manhã e da tarde, visando atender aos horários disponíveis dos entrevistados. Os dados foram coletados de setembro de 2014 a agosto de 2016 no domicílio dos participantes, por meio de entrevistas face a face com entrevistadores treinados, utilizando questionário estruturado. O questionário foi analisado por três juízes (pesquisadores que realizam pesquisas na área de farmacoepidemiologia) para possíveis alterações e adequações. Após essa fase, o instrumento foi pré-testado em um estudo-piloto com 10 participantes para análise da compreensão das perguntas, tendo sido realizadas pequenas alterações. O questionário abordou questões sobre uso, armazenamento e prazos de validade dos medicamentos. Foram abordadas 612 pessoas, das quais 163 recusaram participação, sendo realizadas, portanto, 423 entrevistas.

Todos os participantes entrevistados assinaram o Termo de Consentimento Livre e Esclarecido (TCLE).

\section{Variáveis do estudo}

\section{Variável resposta}

\section{Medicamentos vencidos no estoque caseiro}

A proporção de medicamentos vencidos nos estoques caseiros foi utilizada como variável resposta nos modelos de regressão. Foi calculada da seguinte maneira: número de medicamentos vencidos dividido pelo total de medicamentos nos estoques caseiros.

\section{Variáveis explicativas}

\section{Uso de medicamentos}

Durante a visita em domicílio, o entrevistador analisou o prazo de validade de todos os medicamentos existentes no estoque caseiro, em uso ou não. Foi registrado o nome do princípio ativo de cada medicamento. Com esses dados, foi possível estimar a prevalência de medicamentos vencidos, isto é, a proporção de medicamentos vencidos presentes nos domicílios visitados. Todos os medicamentos foram classificados segundo o sistema de classificação Anatomical Therapeutic Chemical (ATC), index 2017 ${ }^{15}$. Medicamentos isentos de prescrição (MIPs) seguiram a legislação brasileira, a RDC no 138, de 29 de maio de $2003^{16}$. Na classificação ATC, foram considerados na classe "OUTROS" aquelas medicações com frequência inferior a 8.

Foram considerados todos os medicamentos contidos no estoque caseiro dos entrevistados, podendo ser de uso contínuo ou esporádico, em uso atual ou utilizado no passado ${ }^{17,18}$.

\section{Comportamentos relacionados aos medicamentos}

\section{Armazenamento de medicamentos}

Foi considerado armazenamento inadequado se no domicílio fosse identificado pelo menos um dos seguintes itens:
$\checkmark$ Medicamentos expostos diretamente à luz solar;
$\checkmark$ Medicamentos expostos diretamente à umidade;
$\checkmark$ Medicamentos expostos à sujeira (por exemplo, poeira);
$\checkmark$ Medicamentos fora de suas embalagens primárias; 
$\checkmark$ Medicamentos sob alcance de crianças (armazenamento com altura inferior a 1,5 metro);

$\checkmark$ Medicamento armazenado na cozinha ou no banheiro, por serem ambientes com elevada umidade e temperatura;

$\checkmark$ Se termolábeis, armazenamento fora da geladeira ou em sua porta, gavetas e congelador.

Informação sobre descarte de medicamento

Foi perguntado ao entrevistado se ele já havia recebido informação sobre descarte correto de medicamentos.

\section{Guarda de sobra de medicamentos}

O hábito de guardar sobra de medicamentos, vencidos ou não, foi abordado em uma questão do questionário. As opções de resposta eram: sim ou não. Os que responderam afirmativamente foram considerados como pessoas que possuíam o comportamento de acumular medicamentos vencidos ou não vencidos em seu estoque caseiro.

\section{Estratégia para análise de dados}

Inicialmente, as características demográficas e socioeconômicas dos entrevistados foram descritas por meio de proporções para as variáveis categóricas, e por meio da média e desvio-padrão (DP) para a variável contínua. As prevalências e a proporção de medicamentos vencidos foram descritas. A associação entre proporção de medicamentos vencidos e variáveis explicativas foi estimada por meio de modelos lineares generalizados (MLGs). Os MLGs são uma extensão dos modelos clássicos de regressão linear, cuja estimativa de parâmetros é feita pelo método da máxima verossimilhança, e a generalização dos MLGs abre um leque de opções para a distribuição da variável resposta. Assim, os pressupostos básicos de normalidade, linearidade e homocedasticidade não são mais exigidos para a análise dos dados ${ }^{19,20}$. Os resultados são apresentados pela razão de médias aritméticas (RMA), obtida pelo exponencial dos parâmetros gerados pela regressão.

As RMAs brutas e ajustadas, bem como seus respectivos intervalos de confiança de $95 \%$ (IC95\%), foram estimadas. Todas as variáveis que apresentaram associações ao nível de significância menor que 0,20 foram inseridas na análise múltipla, retendo no modelo final apenas aquelas que se mantiveram associadas à variável resposta ao nível de $\mathrm{p}<0,05$. Os dados foram inseridos no Epi Info, versão 7, e as análises foram realizadas no software Stata 12.0 (Stata Corporation, College Station, Estados Unidos).

\section{Aspectos éticos}

O estudo foi aprovado pelo Comitê de Ética em Pesquisa da Universidade Federal de São João del-Rei (UFSJ), campus Centro-Oeste Dona Lindu (CCO), CAAE 30912314.0.0000.5545.

\section{RESULTADOS}

Do total de 423 entrevistados, a maioria (83\%) era do sexo feminino, com idade variando de 18 a 93 anos e média de 58 anos ( $\mathrm{DP}=16)$. A maior parte dos entrevistados $(70 \%)$ possuía até o ensino fundamental completo $(n=294)$. O total de medicamentos encontrados nos estoques caseiros foi de 4,203, com variação de 1 a 44 medicamentos e média de 10 medicamentos por residência $(\mathrm{DP}=5,9)$.

O número de medicamentos vencidos variou de 0 a 15 por residência, com média de 1,05 ( $D P=1,8)$. A prevalência de medicamentos vencidos foi elevada, visto que $45,4 \%$ das residências apresentavam pelo menos um medicamento vencido (Tabela 1). A proporção total de medicamentos vencidos encontrada no presente estudo foi de $10,3 \%$, variando de $0 \%$ a 75\%, com média de 9,4 (DP = 13,9).

No presente estudo, foi observado que os medicamentos de uso esporádico eram os mais prevalentes entre os vencidos, tais como antimicrobianos, analgésicos, antiespasmódicos e 
Tabela 1. Frequência de medicamentos vencidos em estoques caseiros. Divinópolis-MG, Brasil, 2014-2016

\begin{tabular}{ccc} 
Medicamentos vencidos & $\mathbf{N}$ & $\%$ \\
\hline Número de medicamentos vencidos & & \\
\hline Total & $\mathbf{1 9 2}$ & $\mathbf{4 5 , 4}$ \\
\hline Um & 88 & 20,8 \\
\hline Dois & 46 & 10,87 \\
\hline Três ou mais & 58 & 13,71 \\
\hline
\end{tabular}

Tabela 2. Classes de medicamentos vencidos em estoques caseiros, segundo o sistema de classificação Anatomical Therapeutic Chemical (ATC), index 2017¹5. Divinópolis-MG, Brasil, 2014-2016

\begin{tabular}{|c|c|c|}
\hline \multirow{2}{*}{ Classe de medicamentos } & \multicolumn{2}{|c|}{ Vencidos } \\
\hline & $\mathbf{n}$ & $\%$ \\
\hline Antimicrobiano de uso sistêmico & 46 & 10,87 \\
\hline Analgésico & 39 & 9,22 \\
\hline Antiespasmódico e antiemético & 26 & 6,15 \\
\hline Anti-hipertensivo & 26 & 6,15 \\
\hline Anti-inflamatórios não esteroidais (AINEs) & 25 & 5,91 \\
\hline Protetor gástrico & 23 & 5,44 \\
\hline Anti-histamínicos & 20 & 4,73 \\
\hline Suplementos vitamínicos e minerais & 19 & 4,49 \\
\hline Anestésico tópico & 17 & 4,02 \\
\hline Antimicrobiano tópico & 16 & 3,78 \\
\hline Doenças obstrutivas de vias aéreas & 15 & 3,55 \\
\hline Antidepressivo & 14 & 3,31 \\
\hline Anti-inflamatório & 14 & 3,31 \\
\hline Fitoterápico & 14 & 3,31 \\
\hline Antipsicótico & 11 & 2,60 \\
\hline Antigases & 10 & 2,36 \\
\hline Antiparasitário de uso sistêmico & 9 & 2,13 \\
\hline Benzodiazepínicos & 8 & 1,89 \\
\hline Hipolipêmico & 8 & 1,89 \\
\hline Relaxante muscular & 8 & 1,89 \\
\hline Tosse/congestão nasal & 8 & 1,89 \\
\hline Outros $^{d}$ & 62 & 14,66 \\
\hline
\end{tabular}

d: antipsoriáticos; queratolítico; antifúngico de uso sistêmico; antiparasitário de uso tópico; antianêmico; trânsito intestinal/microbiota intestinal; agentes antitrombóticos; antivertiginoso; corticoide; antigotosos; antifúngico tópico; antiosteoporótico; antidiabéticos; antiglaucomatoso e lubrificante ocular; terapia de reposição hormonal/hormônios sexuais/anticoncepcionais; antivirais; cardiovascular

antieméticos (Tabela 2). A presença de MIPs foi associada à maior proporção de medicamentos vencidos nos estoques caseiros, inclusive com gradiente de dose resposta. A classe de protetor gástrico também se mostrou elevada neste estudo, sendo o omeprazol o maior representante dessa classe.

$\mathrm{Na}$ análise do comportamento do entrevistado de guardar medicamentos na residência, foi observada associação com a proporção de medicamentos vencidos. Além disso, a presença 
Tabela 3. Fatores associados à proporção de medicamentos vencidos em estoques caseiros. Divinópolis-MG, Brasil, $2014-2016$

\begin{tabular}{|c|c|c|c|c|}
\hline Variáveis & RMA $^{\mathrm{e}}$ bruta (IC 95\%) & Valor de $p$ & RMA $^{\mathrm{e}}$ ajustada (IC 95\%) & Valor de $p$ \\
\hline Número de medicamentos & $1,04(1,01 ; 1,07)$ & $<0,20$ & $1,02(0,98 ; 1,06)$ & 0,34 \\
\hline \multicolumn{5}{|c|}{ Medicamento isento de prescrição (MIP) } \\
\hline Nenhum & referência & & referência & \\
\hline Um & $2,36(1,36 ; 4,10)$ & $<0,20$ & $2,23(1,27 ; 3,90)$ & $<0,05$ \\
\hline De dois a três & $3,23(1,88 ; 5,55)$ & $<0,001$ & $3,09(1,78 ; 5,35)$ & $<0,05$ \\
\hline Quatro ou mais & $3,60(2,15 ; 6,02)$ & $<0,001$ & $2,74(1,52 ; 4,96)$ & $<0,05$ \\
\hline \multicolumn{5}{|l|}{ Antimicrobiano } \\
\hline Nenhum & referência & & referência & \\
\hline Pelo menos um & $1,80(1,28 ; 2,51)$ & $<0,001$ & $1,62(1,10 ; 2,41)$ & $<0,05$ \\
\hline \multicolumn{5}{|c|}{ Armazenamento correto de medicamentos } \\
\hline Sim & referência & & referência & \\
\hline Não & $1,02(0,59 ; 1,75)$ & 0,95 & $1,40(0,98 ; 1,98)$ & 0,25 \\
\hline \multicolumn{5}{|c|}{ Informação sobre descarte de medicamentos } \\
\hline Sim & referência & & referência & \\
\hline Não & $1,39(0,83 ; 2,31)$ & 0,21 & $0,44(-1,19 ; 0,31)$ & 0,53 \\
\hline \multicolumn{5}{|c|}{ Guarda as sobras de medicamentos } \\
\hline Não & referência & & referência & \\
\hline Sim & $1,56(1,16 ; 2,10)$ & $<0,05$ & $0,27(0,33 ; 0,88)$ & 0,1 \\
\hline
\end{tabular}

eRazão de Média Aritmética (RMA) obtida pelo exponencial do parâmetro resultante do modelo linear generalizado com distribuição gama

de MIPs e antimicrobianos nos estoques caseiros influencia a proporção de medicamentos vencidos, como mostra a Tabela 3.

\section{DISCUSSÃO}

A prevalência de residências com medicamentos vencidos foi elevada, uma vez que, em quase metade $(45,4 \%)$, foram encontrados medicamentos com prazo de validade expirado. Além disso considerando todos os medicamentos encontrados, a proporção de medicamentos vencidos foi de $10,3 \%$. Estudos brasileiros evidenciam que a proporção de medicamentos vencidos varia de 4,2\% a 19,5\% 21,22 , dados que corroboram os achados no presente estudo. Essa elevada prevalência de medicamentos vencidos pode ser justificada por diversos fatores, como a facilidade e a diversidade de fontes de acesso aos medicamentos no Brasil (por exemplo, farmácias privadas, farmácia popular e farmácias do SUS). Os órgãos públicos e privados não apresentam um sistema integrado que permita a comunicação de medicamentos dispensados para cada paciente, não sendo possível, portanto, determinar o que já foi dispensado 23 . A ausência de fracionamento também pode ser uma justificativa, visto que, na maioria das vezes, é necessário dispensar uma quantidade superior àquela que 0 usuário necessita para o tratamento ${ }^{24}$. Assim, há um acúmulo de medicamentos nos estoques caseiros e, consequentemente, vencidos, o que gera gastos desnecessários, além do potencial risco à saúde, visto que a ingestão de medicamentos vencidos pode trazer efeitos indesejados e danosos ao usuário. 
No Brasil, embora o fracionamento seja aprovado e recomendado pela legislação nacional ${ }^{25}$, os medicamentos não são dispensados em quantidades individualizadas para atender às necessidades terapêuticas dos consumidores por falta de vantagens financeiras. O fracionamento é um procedimento que favorece questões econômicas e sanitárias, pois o consumidor tem a possibilidade de comprar somente o número de unidades de medicamentos necessárias para a farmacoterapia. Além de ampliar a racionalização e o acesso da população aos medicamentos, o fracionamento também contribui para a promoção da saúde, uma vez que evita que os pacientes mantenham em sua casa sobras de medicamentos utilizados em tratamentos anteriores. Isso reduz a utilização de medicamentos sem prescrição ou orientação médica, diminuindo o número de intoxicações e de efeitos adversos ${ }^{24}$. Na prática, é observado que o setor industrial farmacêutico possui dificuldade e/ou resistência em aderir ao fracionamento de medicamentos, visto que é necessário haver investimentos em infraestrutura e maquinário para a produção de medicamentos fracionados com a devida segurança e qualidade exigidas pela legislação ${ }^{26}$.

Estudos realizados no México ${ }^{27}$, na Etiópia ${ }^{28}$ e em Uganda ${ }^{29}$ demonstram que os antimicrobianos são os medicamentos mais prevalentes em estoques caseiros. Esse resultado é um fator explicativo para a associação encontrada em ter antimicrobianos no estoque caseiro e maior proporção de medicamentos vencidos no presente estudo. Não obstante a dispensação de antimicrobianos não ser realizada de forma fracionada como prevê a legislação ${ }^{25}$, o controle mais rígido sobre a prescrição e dispensação dessa classe de fármacos ${ }^{30}$ visa à racionalização do uso, evitando, inclusive, que haja sobras do medicamento após o tratamento. Desta forma, a alta prevalência de antimicrobianos em estoques caseiros aponta para o problema da adesão à antibioticoterapia e pode levar aos uso descontrolado e excessivo desses fármacos, fatores importantes no desenvolvimento da resistência aos antimicrobianos ${ }^{28}$.

A associação entre presença de MIPs e maior proporção de medicamentos vencidos nos estoques caseiros pode ser justificada pela facilidade de acesso a esse tipo de medicamento, que estimula a automedicação e facilita o acúmulo e o vencimento nas residências. De fato, uma parcela considerável dos analgésicos (segunda classe mais frequente de medicamentos vencidos) é composta de MIPs. Estudos realizados no Brasil e no exterior identificaram elevada prevalência de analgésicos nos estoques caseiros e de uso por automedicação ${ }^{29,30}$. Isso pode ser justificado pelo hábito de guardar medicamentos principalmente para dor, aguda ou crônica, sendo um fator indutor para automedicação em casos de necessidades.

O comportamento de acumular medicamento reflete no perfil do estoque caseiro, sendo esse hábito comum também em outros países ${ }^{31}$. Neste estudo, foi evidenciado que ele é um fator associado ao vencimento de medicamentos. Diante do exposto, é importante ressaltar que as sobras de medicamentos, vencidos ou não, contidos nos estoques caseiros podem gerar consequências clínicas, econômicas e sociais significativas, como eventos adversos e intoxicações imprevisíveis; prejuízo na qualidade e segurança dos pacientes; aumento das demandas pelos serviços de saúde em geral e impacto econômico negativo para os limitados recursos financeiros destinados ao sistema de saúde. Sendo assim, é preciso orientar a população sobre o uso racional e estimular o descarte correto de medicamentos desnecessários.

O acúmulo de medicamentos de uso crônico vencidos nas residências pode ser um indicador de não adesão à farmacoterapia prescrita, sendo, indiretamente, um parâmetro que merece ser estudado e correlacionado com o controle de parâmetros clínicos preestabelecidos em protocolos clínicos. Outro estudo brasileiro de Baldoni et al. ${ }^{23}$, realizado na mesma cidade da presente pesquisa, identificou que os medicamentos mais devolvidos em unidades de saúde pelos usuários da APS do SUS foram aqueles utilizados para a prevenção e/ou tratamento de doenças crônicas não transmissíveis (DCNT), como hipertensão arterial sistêmica, diabetes mellitus tipo 2, epilepsia e doenças cardiovasculares. Muitas das doenças crônicas são assintomáticas, o que pode contribuir para que o paciente não tenha adesão ao tratamento, impactando no acúmulo de medicamentos vencidos nas residências e no aumento de gastos e recursos para o sistema de saúde, em razão do maior número de consultas e internações por falta de controle dos parâmetros clínicos ${ }^{23}$. 
A alta frequência de protetor gástrico vencido pode estar associada à elevada prevalência de anti-inflamatório não esteroidal (AINE), por ser o primeiro utilizado na prevenção de úlceras e lesões no trato gastrointestinal desencadeados pelo último. $O$ uso inadequado e crônico de omeprazol pode estar associado à má absorção de sais minerais fundamentais ao metabolismo humano, o que pode ocasionar problemas de saúde, como a osteoporose ${ }^{32}$.

É destacada a alta frequência de vencimento de AINEs, o que pode ser justificada, entre outros fatores, pelo perfil etário da população, isto é, adultos e idosos, que são frequentemente acometidos por dores em geral, e pela facilidade de acesso, visto que muitos AINEs (por exemplo, naproxeno, ibuprofeno e cetoprofeno) são MIPs. Os demais AINEs, apesar de a legislação exigir apresentação da prescrição médica, são vendidos livremente na prática das farmácias comunitárias privadas, sem a apresentação da prescrição de um médico ou dentista.

A elevada proporção de medicamentos vencidos é explicada, principalmente, pela presença de classes específicas de medicamentos, como os antimicrobianos e os MIPs. Os resultados evidenciam a necessidade de medidas educativas para a população e para os profissionais de saúde, com intuito de desestimular a manutenção de estoques caseiros de medicamentos, de forma a minimizar desperdícios de recursos dos usuários e dos serviços de saúde, bem como garantir o uso seguro e racional dos medicamentos.

Entre as limitações do estudo é destacado que os resultados não podem ser extrapolados para usuários do sistema privado de saúde, tendo em vista que o estudo foi realizado apenas com usuários da APS do SUS. Em relação aos pontos fortes, vale destacar o rigor metodológico do estudo, a forma de coleta de dados (na residência), que consegue identificar todos os medicamentos utilizados pela família, e a diversidade amostral, que foi realizada de forma aleatória.

Neste estudo, foi observada alta prevalência de residências com armazenamento de medicamentos vencidos, entre os quais se destaca a elevada proporção de fármacos de uso esporádico, como antimicrobianos, analgésicos, antiespasmódicos e antieméticos. Na população estudada, o comportamento de guardar medicamentos na residência esteve associado à proporção de medicamentos vencidos, o que é influenciado pela presença de MIPs e antimicrobianos nos estoques caseiros. Estes achados apontam para a necessidade de se implementarem ações educativas direcionadas aos usuários de medicamentos da APS do SUS, visando à redução do acúmulo de medicamentos em residências e, por consequência, da proporção de medicamentos vencidos nos estoques caseiros como forma de minimizar desperdícios e garantir o uso seguro e racional dos medicamentos.

\section{AGRADECIMENTOS}

Os autores agradecem à Universidade Federal de São João Del-Rei (UFSJ) e ao Conselho Nacional de Desenvolvimento Científico e Tecnológico (CNPq) pelas bolsas concedidas. Além do apoio financeiro da Coordenação de Aperfeiçoamento de Pessoal de Nível Superior (Capes).

\section{REFERÊNCIAS}

1. Luz TCB, Osorio-de-Castro CGS, Magarinos-Torres R, Wettermark B. Trends in medicines procurement by the Brazilian federal government from 2006 to 2013. PLoS One. 2017;12(4):1-13. PMid:28388648.

2. Santos EC, Ferreira MA. A indústria farmacêutica e a introdução de medicamentos genéricos no mercado brasileiro. Rev Nexos Econômicos. 2012;6(2):95-120. http://dx.doi.org/10.9771/1516-9022rene.v6i2.9250.

3. Brasil. Lei no 8.080, de 19 de Setembro de 1990. Dispõe sobre as condições para a promoção, proteção e recuperação da saúde, a organização e o funcionamento dos serviços correspondentes e dá outras providências. Diário Oficial da União, Brasília, 1990.

4. Brasil. Ministério da Saúde. Secretaria de Ciência, Tecnologia e Insumos Estratégicos. Departamento de Assistência Farmacêutica e Insumos Estratégicos. Cuidado farmacêutico na atenção básica. Brasília: Ministério da Saúde; 2014. 108 p. (Caderno 1: Serviços farmacêuticos na atenção básica à saúde).

5. Organización Mundial de la Salud - OMS. Promoción del uso racional de medicamentos: componentes centrales. Ginebra: OMS; 2000. 
6. Ryan TP, Morrison RD, Sutherland JJ, Milne SB, Ryan KA, Daniels JS, et al. Medication adherence, medical record accuracy, and medication exposure in real- world patients using comprehensive medication monitoring. PLoS One. 2017;12(9):1-19. http://dx.doi.org/10.1371/journal.pone.0185471. PMid:28957369.

7. Faria HTG, Santos MA, Arrelias CCA, Rodrigues FFL, Gonela JT, Teixeira CR S, et al. Adesão ao tratamento em diabetes mellitus em unidades da Estratégia Saúde da Família. Rev da Esc Enferm. 2014;48(2):254-60.

8. Mirza N, Ganguly B. Utilization of medicines available at home by general population of rural and urban set up of Western India. J Clin Diagn Res. 2016;10(8):FC05-09. http://dx.doi.org/10.7860/ JCDR/2016/20600.8298. PMid:27656460.

9. Chinnaiyan S, Narayana SNV, Nanjappa VP. Adherence to antiepileptic therapy in adults. J Neurosci Rural Pract. 2017;8(3):417-20. http://dx.doi.org/10.4103/jnrp.jnrp_392_16. PMid:28694623.

10. Ekedahl ABE. Reasons why medicines are returned to Swedish pharmacies unused. Pharm World Sci. 2007;28(6):352-8. http://dx.doi.org/10.1007/s11096-006-9055-1. PMid:17221141.

11. Law AV, Sakharkar P, Zargarzadeh A, Tai BWB, Hess K, Hata M, et al. Taking stock of medication wastage: unused medications in US households. Res Social Adm Pharm. 2015;11(4):571-8. http://dx.doi. org/10.1016/j.sapharm.2014.10.003. PMid:25487420.

12. Pereira JR, Soares L, Hoepfner L, Kruger KE, Guttervil ML, Tonini KC, et al. Riscos da Automedicação? Tratando o problema com conhecimento. Joinville: Univille. 2008. p. 1-20.

13. Kusturica MP, Tomas A, Tomic Z, Bukumiric D, Corac A, Horvat O, et al. Analysis of expired medications in serbian households. Zdr Varst. 2016;55(3):195-201. http://dx.doi.org/10.1515/sjph-2016-0025.

14. Sistema de informática do Sistema Único de Saúde - DATASUS. População residente em Divinópolis. Brasília: Ministério da Saúde; 2012.

15. Organização Mundial de Saúde - OMS. Anatomical Therapeutic Chemical (ATC), index. 2016. [citado em 2017 Jan 25]. Disponível em: https://www.whocc.no/atc_ddd_index/.

16. Brasil. Ministério da Saúde. Agência Nacional de Vigilância Sanitária - ANVISA. Resolução - RDC n 138, de 29 de maio de 2003. Diário Oficial da União, Brasília, 2003. p. 37.

17. Hussain A, Malik M, Iram R. Medicine storage trends \& practices: a literatura review. J Appl Pharm. 2017;9(1):1-14. http://dx.doi.org/10.21065/19204159.9.1.1.

18. De Bolle L, Mehuys E, Adriaens E, Remon JP, Van Bortel L, Christiaens T. Home medication cabinets and self-medication: a source of potential health threats? Ann Pharmacother. 2008;42(4):572-9. http://dx.doi. org/10.1345/aph.1K533. PMid:18364405.

19. McCullagh P, Nelder J. Generalized linear models. Atlanta: Chapman Hall; 1989. http://dx.doi. org/10.1007/978-1-4899-3242-6.

20. Nelder W, Wedderburn RWM. Generalized linear models. J R Stat Soc [Ser A]. 1972;135(3):370-84. http:// dx.doi.org/10.2307/2344614.

21. Fanhani HR, Lorenson L, Karoline P. Avaliação domiciliar da utilização de medicamentos por moradores do Jardim Tarumã, Município de Umuarama - Pr. Arq Ciên Unipar. 2006;10:127-31.

22. Tsiligianni IG, Delgatty C, Alegakis A, Lionis C. A household survey on the extent of home medication storage. A cross-sectional study from rural Crete, Greece. Eur J Gen Pract. 2012;18(1):3-8. http://dx.doi.or g/10.3109/13814788.2011.604674. PMid:21879836.

23. Baldoni AO, Gontijo JD, Silva VKC, Fernandes MR, Alvim CP, Ferreira SM, et al. Armazenamento e descarte de medicamentos: estratégia educativa e perfil de medicamentos descartados. Rev eletrônica extensão UFSC. 2015;12:48-61.

24. Murakami I, Ribeiro LM No, Silva AM. A importância do fracionamento de medicamentos para o SUS: um estudo piloto. Rev Bras Farm. 2012;93(2):221-4.

25. Brasil. Ministério da Saúde. Agência Nacional de Vigilância Sanitária - ANVISA. Resolução - RDC n 80, de 11 de maio de 2006. Diário Oficial da União, Brasília; 2006; p. 27.

26. Oliveira ND. Estudo da implementação do fracionamento de medicamentos no Brasil: aspectos regulatórios, acesso e uso racional [dissertação]. Porto Alegre: Faculdade de Farmácia, Universidade Federal do Rio Grande do Sul; 2009.

27. Gracia-Vásquez SL, Ramírez-Lara E, Camacho-Mora IA, Cantú-Cárdenas LG, Gracia-Vásquez YA, EsquivelFerriño PC, et al. An analysis of unused and expired medications in Mexican households. Int J Clin Pharm. 2015;37(1):121-6. http://dx.doi.org/10.1007/s11096-014-0048-1. PMid:25492270.

28. Wondimu A, Molla F, Demeke B, Eticha T, Assen A, Abrha S, et al. Household storage of medicines and associated factors in Tigray Region, Northern Ethiopia. PLoS One. 2015;10(8):1-9. http://dx.doi.org/10.1371/ journal.pone.0135650. PMid:26275057. 
29. Ocan M, Bbosa GS, Waako P, Ogwal-Okeng J, Obua C. Factors predicting home storage of medicines in Northern Uganda. BMC Public Health. 2014;14(1):650. http://dx.doi.org/10.1186/1471-2458-14-650. PMid:24964746.

30. Brasil. Ministério da Saúde. Agência Nacional de Vigilância Sanitária - ANVISA. Resolução RDC n 20, de 5 de maio de 2011. Dispõe sobre o controle de medicamentos à base de substâncias classificadas como antimicrobianos, de uso sob prescrição, isoladas ou em associação. Diário Oficial da União [Internet], Brasília; 2011 [citado em 2018 Dez 8]. Disponível em: http://www.anvisa.gov.br/sngpc/Documentos2012/ RDC 20 2011.pdf

31. Bashaar M, Thawani V, Hassali MA, Saleem F. Disposal practices of unused and expired pharmaceuticals among general public in Kabul. BMC Public Health. 2017;17(1):45. http://dx.doi.org/10.1186/s12889-0163975-z. PMid:28061902.

32. Hipólito P, Rocha BS, Oliveira FJAQ. Perfil de usuários com prescrição de omeprazol em uma Unidade Básica de Saúde do Sul do Brasil: considerações sobre seu uso racional. Rev Bras Med Fam Comunidade. 2016;11(38):1-10. http://dx.doi.org/10.5712/rbmfc11(38)1153. 